\title{
Going beyond the TRIGR study: The environment and the type 1 diabetes in Sardinia
}

\author{
Carla Mannu and Marco Songini* \\ Department of Internal Medicine Diabetes Unit, Azienda Ospedaliera 'Brotzu', Cagliari, Italy
}

Type 1 diabetes mellitus (T1D) results from an autoimmune destruction of insulin-producing $\beta$ cells, with lack of insulin. T1D is one of the most chronic disease of childhood, increases mortality and reduces quality of life. Newly significant technological advances have been achieved in the treatment and quality of life in diabetics but the causes are still uncertain, so its prevention is still far away. Genetic factors are relatively well known, even most people genetically predisposed to T1D do not develop it. The genetic factors alone do not explain the increased risk of T1D, sharply increased over the last 40 years in Sardinia, with the second highest risk in the world after Finland [1]. It is still not known why some people develop T1D although it is agreed that genetic, non genetic and probably epigenetic environmental factors contribute to the disease. The environmental factors are probably very important for the development and the increase of T1D. The epigenetic factor possible interrelationships are to be cleared at most.

All these factors make the Island an ideal region for investigation of T1D. Consequently, several studies have been carried out in the Island toward the aetiopathogenesis of T1D [1]. As the primary prevention trials, we have participated in the TRIGR study (Trial to Reduce IDDM in the Genetically at Risk,), the first T1D primary prevention study (double blind trials) in the world that started in 2002 [2].

The hypothesis of the study was that the early exposure to cow's milk could have accelerated the destruction of $\beta$ cells in genetically predisposed individuals and that the weaning with an extensively hydrolysed formula could decrease the risk of T1D in young children. In Sardinia, the TRIGR study was conducted at the Diabetes Center of Brotzu Hospital in Cagliari, one of the 77 centres that had participated across Europe, Australia, Canada and the United States, with over 2,800 children recruited. It has been proposed to Sardinian patients with T1D the genetic screening of their children. Newborn infants, with a defined human leukocyte antigen (HLA) genotypes, were recruited between May 2002 and January 2007 and then followed up until the youngest participant reached 10 years of age. Enrolled infants received, in addition to breastfeeding (breastfeeding was always encouraged), the hydrolysed casein formula or cow's milk for the first 6-8 months of life. The children, in the first year, underwent checkups every three months and thereafter annually until the completion of the study. To best assess the different stages of diabetes, the concentration of antiislet antibodies (early stage of diabetes) and the basal blood glucose level, glycated haemoglobin (HbA1c) or load curve (OGTT) (clinical diabetes) have been determined.

We are now in the 15th year of TRIGR study and close to the end: out of the 17 children of our center, only 4 developed diabetes between
2 and 5 years. In December 2016 we completed the study visits. Enrolled children will still be followed up online: they will be asked to keep us informed on the eventual occurrence of the clinical disease even in their family members. The final result of the TRIGR Study was published on 2 Jan 2018 in the Journal of the American Medical Association (JAMA): an extensively hydrolyzed casein formula during infancy did not result in a reduction in the incidence of type 1 diabetes compared to regular and the intact cow's milk-based formula [2]. As a result, the question is still open: "What are the environmental factors that contribute to increasing the risk of diabetes?"

The T1D in Sardinia still remains an "enigma”. Recent studies have linked the elevated presence of Micobacterium Avium Paratubercolosis (MAP, a bacterium frequent present in cow's milk) to the onset of T1D in the Sardinian population [3]. The unique geochemistry of Sardinia with the particular concentration of heavy metals has suggested another triggering factor such as exposure to heavy metals, already associated with the development of other autoimmune diseases. In our search for the correlation between the incidence of T1D and heavy metals (performed in collaboration with the University Geology group led by Paolo Valera) a slight negative correlation ( $\mathrm{r}=-0.332 ; p$ $=0.0002$ ) between zinc and T1D was found [4]. These results would suggest a protective role of zinc on the development of the disease, and its deficiency could be a triggering cofactor. A negative association between T1D risk and ultraviolet B (UV-B) solar irradiation has been suggested $[5,6]$. We conducted an ecological analysis to assess the possible relationships between UV-B radiation levels and T1D incidence in Sardinia (always with the geology team of Paolo Valera). A standardized algorithm based upon the solar constant and the latitude of each municipality has been employed to calculate the amount of total solar irradiance. UV-B radiation during the winter solstice for each Sardinian municipality was then calculated. This value was adjusted to the annual average of cloudiness and to the percentage of direct solar irradiation of the territory. T1D incidence data were obtained through the Sardinian Diabetes Registry. The relationship between UV-B radiation and T1D incidence in Sardinia was assessed through a simple correlation analysis. A mild negative correlation $(\mathrm{r}=-0.154 ; p=$ 0.002 ) was obtained between UV-B radiation and T1D incidence [7]. A protective effect (even weak) of UV-B irradiance in T1D and/or a role of vitamin $\mathrm{D}$ deficiency on T1D risk was suggested by many authors

Correspondence to: Marco Songini, Department of Internal Medicine Diabetes Unit, Azienda Ospedaliera 'Brotzu', Cagliari, Italy, E-mail: marcosongini@aob.it

Received: January 19, 2018; Accepted: January 22, 2018; Published: January 30, 2018 
and our results are consistent with this hypothesis (protective role of sun exposure?). From this we hypothesize that the incidence of T1D could be influenced by exposure to multiple risk factors such as: MAP, common viruses, heavy metals, Zinc deficiency and solar irradiation which together would participate in raising the risk of incidence of diabetes. The search for the "puzzle" is still open: what environmental factors actually are involved in the disease and its prevention?

\section{Acknowledgement}

Paolo Valera (Department of Civil-Environmental Engineering and Architecture - University of Cagliari, Italy) and Leonardo A. Sechi (Department of Biomedical Sciences, University of Sassari, Italy).

\section{References}

1. Songini M, Mannu C, Targhetta C, Bruno G (2017) Type 1 diabetes in Sardinia: facts and hypotheses in the context of worldwide epidemiological data. Acta Diabetol 54(1): 9-17. [Crossref]
2. Writing Group for the TRIGR Study Group JAMA (2018) Effect of Hydrolyzed Infant Formula vs Conventional Formula on Risk of Type 1 Diabetes: The TRIGR Randomized Clinical Trial JAMA 319(1): 38-48. [Crossref]

3. Niegowska M, Paccagnini D, Mannu C, Targhetta C, Songini M, et al (2016) Recognition of ZnT8, Proinsulin, and Homologous MAP Peptides in Sardinian Children at Risk of T1D Precedes Detection of Classical Islet Antibodies J Diabetes Res 2016(2016) doi: 10.1155/2016/5842701. [Crossref]

4. Valera P, Zavattari P, Sanna A, Pretti S, Marcello A et al (2015) Zinc and Other Metals Deficiencies and Risk of Type 1 Diabetes: An Ecological Study in the High Risk Sardinia Island. PLoS One 10(11): e0141262. [Crossref]

5. Staples JA, Ponsonby AL, Lim LLY, McMichael AJ (2003) Ecologic analysis of some immune-related disorders, including type 1 diabetes, in Australia: latitude, regiona ultraviolet radiation, and disease prevalence Environ Health Perspect 1114: 518-523. [Crossref]

6. Mohr SB, Garland CF, Gorham ED, Garland FC (2008) The association between ultraviolet B irradiance, vitamin D status and incidence rates of type 1 diabetes in 51 regions worldwide. Diabetologia 51(8): 1391-1398. [Crossref]

7. Songini (2017) Diabetes and Its Complications (ICDC-2017). November 2-4, Baltimore, USA.

Copyright: (C2018 Mannu C. This is an open-access article distributed under the terms of the Creative Commons Attribution License, which permits unrestricted use, distribution, and reproduction in any medium, provided the original author and source are credited. 